\title{
Entorno familiar y crianza del menor Wayuü trabajador del basurero de Riohacha-Colombia*
}

\author{
Family environment and upbringing of the younger worker Wayuu \\ from the dustbin of Riohacha - Colombia
}

Recibido 23. 10. $2018 \bullet$ Arbitrado 19. 11. $2018 \bullet$

Aprobado 15.12. 2018

Artículo se deriva de la investigación "Entorno familiary prácticas decrianza del menor trabajador del basurero de Riohacha", realizada para optar al título de Magister en Desarrollo integral de niños y adolescentes, Facultad de Psicología Universidad Cooperativa de Colombia Seccional Santa Marta, año 2016. Se realizó con el apoyo financiero de la Universidad de la Guajira y el apoyo de académico de la Maestría en Desarrollo Integral de niños y adolescentes, de la facultad de Psicología de la Universidad Cooperativa de Colombia Seccional Santa Marta.

** Magister en Desarrollo integral de niños y adolescentes, Docente Universidad de la Guajira, Colombia, anreme79@hotmail.com, ORCID: 00000002-8808-3060

*** Magister en Desarrollo integral de niños y adolescentes, Docente Universidad de la Guajira, Colombia, belpinto0714@gmail.com, ORCID: 00000002-6848-2929

**** PhD. en Psicología Universidad del Norte- Colombia, Docente Facultad de Psicología, Universidad Cooperativa de Colombia, Santa Marta, Colombia, sara.zabarain@campusucc.edu.co, ORCID: 0000-0003-1685-0715

\section{Resumen}

El artículo presenta resultados de la investigación descriptiva transversal orientada a caracterizar el entorno familiar y prácticas de crianza en una muestra de las madres de los menores trabajadores del basurero de Riohacha-Guajira, Colombia. La población estuvo constituida por 11 madres de la etnia Wayuü que habitan alrededor del basurero y trabajan reciclando, incluyendo sus hijos. Se recolectó información mediante ficha sociodemográfica y encuesta sobre prácticas de crianza con validez interna y de contenido. Se concluye que el modelo de crianza está arraigado, heredado de la cultura ancestral wayuü, predominando el matriarcado. La madre se preocupa por la protección y el cuidado físico, pero no posee los conocimientos adecuados para la regulación afectiva de sus hijos.

Palabras claves: Entorno familiar; Prácticas de crianza; Afecto; Menor trabajador; Wayuü.

\section{Abstract}

This cross-sectional descriptive research characterizes the family environment and parenting 
practices of the mother of the minor worker of the Riohacha-Guajira garbage dump, Colombia. The population was composed of 11 Wayuü mothers who live around the dump and work recycling, including their children. Information was collected through a sociodemographic record and a survey about parenting practices with internal validity and content. It is concluded that the model of upbringing is rooted, inherited from the ancestral Wayuü culture, with matriarchy predominating. The mother cares about protection and physical care, but does not possess the adequate knowledge for affective regulation of her children.

Keywords: Family environment; Parenting practices; Affected; Minor worker Wayuü.

\section{Introducción}

En el entorno familiar, que constituye todos los elementos, factores y principios rectores que influyen en la familia, cada uno de los miembros que hacen parte de este va adoptando un rol social, que depende del contexto socio económico, momento, cultura, entre otros aspectos que conllevan a que el entorno sea cambiante. De acuerdo con Coria, Jasso, Paz y Nájera (2012) dentro de esos principios rectores que hacen parte del entorno familiar y pueden regir el comportamiento de las familias, se destacan las prácticas de crianza. Así de este modo, entorno familiar y prácticas de crianza están en estrecha relación en el ejercicio de la crianza, cuidado y educación del menor.

Las prácticas de crianza hacen parte del entorno familiar y son lineamientos o reglas que los padres adoptan con los hijos, teniendo en cuenta la internalización de los modelos de crianza generacionales y aspectos relacionados con la cultura. También se puede entender que "las prácticas de crianza "son los pensamientos, creencias y preocupaciones que a través del tiempo han modulado la relación de los adultos con los niños" (Torres, 2009, p. 25); y los cuales han enfrentado cambios debido a los modelos que adoptan los padres de acuerdo a los diversos tipos de familia.

Dentro de la relación entorno familiar y prácticas de crianza hay dos aspectos importantes, la familia y la cultura, que influyen en la manera de como los padres deciden criar a sus hijos; a estos aspectos se suman el de la pobreza y la desesperanza, que en algunos casos confluyen en la posibilidad de que un menor acceda de manera temprana al trabajo.

Según informe de la OIT (2017, p.9) "En el mundo hay 152 millones de niños-64 millones de niñas y 88 millones de niños- en situación de trabajo 
infantil; es decir casi 1 de cada 10 niños en todo el mundo (...). 73 millones de niños en términos absolutos-realizan trabajos peligrosos que directamente ponen en riesgo salud, su seguridad o su desarrollo moral". Además del riesgo y la afectación en las áreas anteriores, otros aspecto importantes a contemplar serían el desarrollo social, el desarrollo cognitivo y el desarrollo académico, debido a que las horas empleadas en la dedicación laboral disminuyen el tiempo que podrían dedicarle a compartir las actividades al aire libre con compañeros o amigos, o el tiempo en el que podrían asistir a una jornada en el colegio. Las prioridades de los niños estarían dadas al acompañamiento del ingreso económico de la casa o de la familia, antes de otras actividades propias de la infancia.

De igual forma, según los datos del DANE (2016), la cifra nacional de trabajo infantil en Colombia en el último trimestre de 2016 fue de un 7,8\% del total de la población del país. Comparando con la cifra de ese mismo periodo de 2015 que fue de $9,1 \%$, tuvo una reducción de 1,3\% con relación a los rangos etarios de la población entre los 5 y los 14 años de edad, con una dedicación aproximada de 15 horas semanales. Como se puede apreciar, Colombia está muy por debajo de los promedios y proporciones mundiales, pero es preocupante la situación dado que lo ideal es que estas cifras sean cero.

Otro aspecto que se suma a la desprotección del menor y la vulneración de sus derechos, teniendo en cuenta la anterior preocupación, es que en la actualidad el Estado y la sociedad han ido perdiendo su rol de sujetos activos, para convertirse prácticamente en entes pasivos y poco sensibles ante la dramática situación que atraviesan los menores trabajadores, descuidando la necesidad de reconocerles y garantizarles una protección especial y sus derechos (González y Pimienta, 2004). Por lo tanto, lo estipulado en la Ley 1098 de 2006, parece quedar en el papel, porque poco se hace valer los derechos consagrados en el Código del Menor y en la misma Constitución Política.

De igual forma al tener en cuenta los aspectos culturales, cabe resaltar que Colombia, es país politécnico y pluricultural, en donde existen diversas regiones con asentamientos históricos étnicos, pero que comparten los territorios con otras etnias. Esta diversidad ha permitido que todos los grupos étnicos de alguna forma se influencien unos a otros de manera significativa.

Este este es el caso de la Guajira, un departamento que, a lo largo de toda su extensión, ha sido ocupado desde mucho tiempo por la etnia Wayuü y por otras etnias con menos influencia en este territorio, como son los Kogui, Wiwa y Tayronas. También este departamento ha tenido asentamientos de poblaciones culturalmente diferentes a las indígenas, como alijunas o ariju- 
nas, porque no pertenecen a la cultura wayuü; esta población son los blancos, afrodescendientes y mestizos.

Como es usual, los pueblos o culturas más avanzados o fuertes terminan imponiendo sus costumbres, su manera de comportarse y de expresarse, pero en el caso del departamento de la Guajira, la cultura wayuu conserva su modo de vida, su lengua y su modo o prácticas de crianza; e, infortunadamente, entre algunos modelos que han adoptado de los alijunas, se encuentra el trabajo infantil.

En Riohacha, se ha extendido el trabajo infantil en niños y niñas de distintas edades, los cuales realizan diversas actividades económicas informales. Esto se debe a situaciones de pobreza extrema y al desplazamiento por la violencia. Esta dinámica familiar converge en elementos escasos de cuidado y protección a los menores, constituyéndose en factores de riesgo afectivos, físicos y emocionales para el desarrollo integral de los niños Wayuü, sobre todo a los que se encuentran ubicados en barrios marginales y sectores periféricos de la ciudad.

Específicamente se puede mencionar el caso de las familias que habitan en los alrededores del basurero de Riohacha, caracterizadas por pertenecer a la etnia Wayuü, las cuales comparten muchos patrones culturales de los alijunas, sin embargo, conservan su identidad, pero viven en condiciones sociales y económicas reprobables dependiendo del subempleo o trabajo no digno, en la mayoría de estas existe la figura del menor trabajador.

A partir de lo expuesto anteriormente, se planteó esta investigación, por cuanto el trabajo infantil trae, a su vez, una serie de consecuencias entre las que se cuentan la desescolarización, la baja calidad en el aprendizaje, los peligros para la integridad y en la salud, en el desarrollo físico, psíquico, emocional y social, la desintegración familiar y riesgo de recurrir a caminos de mayor peligro como la violencia y la drogadicción, además de desconocer los riesgos y peligros a los cuales están expuestos; siendo el objeto de estudio de esta investigación caracterizar el entorno familiar y las prácticas de crianza que asume la madre del menor trabajador de la etnia Wayuü de la ciudad de Riohacha -La Guajira.

En cuanto a las características del entorno familiar, se tomó en cuenta los aspectos del contexto familiar como salud, actividades económicas, tipos de viviendas, nivel educativo y tipos de familias, y las prácticas de crianza que adoptan las madres de los menores trabajadores del basurero de Riohacha, con relación al cuidado, las relaciones afectivas, los estilos y la percepción en la crianza. También se indago sobre el concepto de trabajo en menores que 
tienen los padres de familia, ya que es un tema que difícilmente puede ser abordado debido a aspectos culturales de la región de La Guajira y sus etnias indígenas.

\section{Método}

El estudio fue de tipo exploratorio-descriptivo, debido a que el tema de entorno familiar y prácticas de crianza adoptadas por madres de la etnia Wayuu de menores trabajadores han sido poco estudiados, lo cual, considerando la perspectiva empleada en el estudio, prepara el terreno para nuevos estudios relacionados.

\section{Participantes}

La población objeto de investigación estuvo constituida por 11 madres de familias de la etnia Wayuü que habitan en los alrededores del basurero de la ciudad de Riohacha. Este tamaño pequeño de la población permitió utilizar un muestreo no probabilístico intencional, analizándose los once casos de las madres de familias wayuü que habitaban en esa localidad.

Para seleccionar la muestra se tuvieron en cuenta los siguientes criterios: familias censadas el ministerio de trabajo y casa de justicia, madres pertenecientes a familias de la etnia Wayuü; madres con hijos menores trabajadores y que el trabajo que desempeñaran era el reciclaje en el basurero de Riohacha.

\section{Instrumentos}

Para la recolección de datos se empleó una ficha sociodemográfica y encuesta. La ficha sociodemográfica permite medir los siguientes aspectos: datos familiares, aspectos contextuales, salud, actividades económicas, tipo de vivienda, nivel educativo, tipo de familia. En cuanto a la identificación de las prácticas de crianza adoptadas por la madre de la etnia Wayuu del menor trabajador de Riohacha se utilizó una encuesta que tuvo en cuenta los siguientes aspectos: el cuidado, las relaciones afectivas, los estilos de crianza y la percepción en la crianza.

Tanto en la ficha, como la encuesta se consideraron preguntas cerradas, con respuestas múltiples, permitiendo a las madres encuestadas tener varias posibilidades, convirtiéndose así en un instrumento completo que permitió realizar un informe detallado de la población en estudio en cuanto a la descripción de las prácticas de crianza en el menor trabajador del basurero de la ciudad 
de Riohacha - La Guajira. En el momento de la aplicación de los instrumentos se contó con la presencia de una persona conocedora del lenguaje de la etnia Wayuü; así mismo las investigadoras desarrolladoras de esta investigación son nativas (arijunas) de esta región de la Guajira-Colombia y conocedoras de las costumbres de esta población. Los jueces evaluadores de los instrumentos, igualmente, son conocedores de las costumbres de esta población.

El diseño de los instrumentos de recolección de información se hizo teniendo en cuenta las variables Entorno familiar y Practicas de crianza. El entorno familiar se entiende como la serie de principios rectores que guían el comportamiento familiar y la situación que se vive en su interior, donde cada miembro va adoptando un rol social, según el contexto, momento, cultura y otros aspectos que hacen que sea cambiante con el tiempo, época, situaciones, lugar, cultura, entre otros factores, modificando a su vez esos principios (Coria et al., 2012); y las prácticas de crianza hacen referencia a los pensamientos y creencias que tienen los adultos acerca de cómo educar a sus hijos (Torres, 2009). Para el entorno familiar se tuvieron en cuenta los indicadores: datos familiares, aspectos contextuales, salud, actividades económicas, tipo de vivienda y nivel educativo. Para las prácticas de crianza se tuvieron en cuenta los indicadores: cuidado, relaciones afectivas, estilos de crianza y percepción en la crianza.

Estos instrumentos se validaron de tres maneras: a) se realizó previamente una prueba piloto con sujetos que reunían características similares a la población sujeto de estudio de esta investigación; b) se realizó la validez de contenido por medio de la evaluación de jueces expertos; y se realizó c) validez interna mediante el cálculo del Coeficiente de Confiabilidad de Alfa de Cronbach para todos los 48 ítems o preguntas que contiene este instrumento, obteniendo el valor de 0,519. Para el análisis estadístico se utilizó el SPSS y Excel.

\section{Procedimiento}

Para el manejo ético de este estudio se utilizó la firma de consentimientos informados de las madres de los menores trabajadores. Para la lectura de los consentimientos informados y de toda la información e instrumentos, se utilizó una interprete conocedora del lenguaje de la etnia Wayuü, para el total entendimiento de toda la documentación teniendo en cuenta la diferencia en el idioma de las participantes. 
Además, se tuvo en cuenta los aspectos legales, y las políticas públicas y culturales, pertenecientes a la etnia Wayuü, siguiendo lo estipulado referente al resguardo y confidencialidad de datos suministrados por los informantes en la Ley 1581 de 2012 en cuanto a la reserva de nombres u otros aspectos que puedan identificar a las participantes.

\section{Resultados}

\section{Características del entorno familiar}

A continuación, se presentan los resultados obtenidos de la aplicación de los instrumentos. En primera instancia se hablará del entorno familiar correspondiente a los aspectos representados en el contexto y las actividades de la familia, específicamente a lo concerniente a las actividades que realiza la madre del menor trabajador. Estos resultados se obtuvieron a través de la ficha sociodemográfica.

En la Tabla 1, se puede observar que el rango etario predominante fue de 26 a 46 años, representando el 56\% de las madres, siendo el promedio de la edad de las madres participantes de 33,5 años con desviación típica de 11,9 años; lo que indica que, las madres en general, se encuentran entre la adultez joven y mediana adultez. En esta etapa se fortalecen las relaciones con los hijos (Viguer y Serra, 1996) y, además, se desarrollan las competencias personales de la etapa del logro (Erikson, 2000; Schaie y Willis 2016), donde el conocimiento adquirido es útil por lo que se puede lograr con él, en este caso mantener la familia y criar los hijos.

Se observa que existe un número promedio 5,64 de hijos por cada madre. Menos de 5 hijos es el número más representativo $(45,4 \%)$, mientras que los otros rangos, de 5 a 9 hijos y de más de 9 de hijos, tuvieron una participación menor en cada rango (Tabla 1). Esto sugiere una tendencia de las madres del estudio hacia las familias numerosas. Igualmente, se observó que más de la mitad de las madres participantes de la investigación, consideraron que, ellas y sus familias han sido víctimas de desplazamiento forzado (grupos al margen de la ley) (54,6\%), lo que ha repercutido en disminuir las oportunidades de establecerse permanentemente en un solo hábitat. 
Tabla N 1: Características sociodemográficas de las madres participantes.

\begin{tabular}{lccc}
\hline & Rango etario por años & Frecuencia & $\%$ \\
\cline { 2 - 4 } Edad & $<26$ & 3 & $27 \%$ \\
\cline { 2 - 4 } & 26 a 49 & 6 & $55 \%$ \\
\cline { 2 - 4 } & $>49$ & 2 & $18 \%$ \\
\cline { 2 - 4 } Número de hijos & Rango & Frecuencia & $\%$ \\
\cline { 2 - 4 } & $<5$ & 5 & $45,4 \%$ \\
\cline { 2 - 4 } & 5 a 9 & 3 & $27,3 \%$ \\
\hline Victimización & $<9$ & 6 & $27,3 \%$ \\
pyor desplazamiento & $\mathrm{Si}$ & 5 & $45,6 \%$ \\
\hline Total & No & 11 & $100 \%$ \\
\hline
\end{tabular}

Fuente: Propia

Se puede evidenciar que la mayoría de las madres participantes del estudio, consideraron que sus ingresos son suficientes para cubrir sus necesidades básicas de alimentación (63,6\%); sin embargo, se hace la acotación que en el momento de diligenciar esta ficha sociodemográfica se observó que las familias viven en condiciones de vivienda poco saludables, y existe desnutrición en algunos de sus hijos y tampoco cubren totalmente la necesidad de educación (Tabla 2). Se podría inferir que existe en ellas una buena capacidad de adaptación a las diversas condiciones de su contexto, algo propio de la etnia Wayuu.

Tabla 2: Necesidades básicas que cubren las familias

\begin{tabular}{lcccccc}
\hline Alcanza & \multicolumn{2}{c}{ Alimentación } & \multicolumn{2}{c}{ Educación } & \multicolumn{2}{c}{ Vivienda } \\
\hline & $\mathbf{n}^{\mathbf{o}}$ & $\mathbf{\%}$ & $\mathbf{n}^{\mathbf{o}}$ & $\mathbf{\%}$ & $\mathbf{n}^{\mathbf{o}}$ & $\mathbf{\%}$ \\
\hline Si & 7 & $63,6 \%$ & 9 & $81,8 \%$ & 8 & $72,7 \%$ \\
\hline No & 4 & $36,4 \%$ & 2 & $8,2 \%$ & 3 & $27,3 \%$ \\
\hline Totales & $\mathbf{1 1}$ & $\mathbf{1 0 0} \%$ & $\mathbf{1 1}$ & $\mathbf{1 0 0} \%$ & $\mathbf{1 1}$ & $\mathbf{1 0 0 \%}$ \\
\hline
\end{tabular}

Fuente: Propia

Se puede observar que el tipo de familia predominante en la comunidad que habita en los alrededores del basurero de Riohacha es mononuclear; 10 de las 11 familias son de ese tipo (91\%), solo una es de madre-solterísimo (Tabla 3). Por lo tanto, existe la tendencia en esta población del estudio hacia la familia tradicional. 
Tabla 3: Tipos de familia

\begin{tabular}{lcc}
\hline \multicolumn{1}{c}{ Opciones } & $\mathbf{N}^{\circ}$ & $\mathbf{\%}$ \\
\hline Madre-solterísimo & 1 & $9 \%$ \\
Familia mononuclear & 10 & $91 \%$ \\
Otros tipos de familia & 0 & $0 \%$ \\
Total & $\mathbf{1 1}$ & $\mathbf{1 0 0} \%$ \\
\hline
\end{tabular}

Fuente: Propia

De manera general se puede decir que el entorno familiar del menor trabajador, es un contexto que presenta carencia y dificultades. La familia sigue siendo una familia tradicional conformada por el padre, la madre y los hijos. La percepción de las madres es que los ingresos captados por la familia son suficientes de acuerdo a sus tradiciones, pero se observa un ambiente de pobreza y carencias alimentarias. Los ingresos de las familias están relacionados con la actividad de recolección en el basurero, el diseño de artesanías y otros trabajos esporádicos como el lavado e carros por la calle. Tienen vivienda propia adaptada a sus tradiciones con poco o escaso cubrimiento de servicios como agua y luz; perciben que los organismos del estado colombiano no les brindan un apoyo suficiente. A esto se le suma la presencia de grupos armados al margen de la ley en la región que les limita el acceso a otros servicios.

\section{Características de las prácticas de crianza}

Para la identificación de las prácticas de crianza del menor trabajador del basurero de Riohacha se aplicó como instrumento una encuesta sobre prácticas de crianza dirigida a padres (o madres), la cual se aplicó en este caso a las madres de familia. La variable prácticas de crianza fue abordada desde las dimensiones: cuidado, relaciones afectivas con los padres, estilos de crianza y percepción de la crianza. A continuación se exponen algunos resultados con relación al cuidado.

Se puede observar que la mayoría de las madres participantes en el estudio respondieron haber dado a sus hijos leche materna hasta los 12 meses de edad, y este fue el alimento básico durante 6 meses. La tradición en este grupo de madres es hacia la alimentación tradicional (Tabla 4). 
Tabla 4: Edad de finalización y duración de lactancia materna como alimento básico.

\begin{tabular}{lcc}
\hline Edad de finalización & Duración/Meses como alimento básico & \% \\
\hline 12 meses & 6 & $55 \%$ \\
13 a 18 meses & 1 & $9 \%$ \\
19 a 24 meses & 4 & $36 \%$ \\
Total & $\mathbf{1 1}$ & $\mathbf{1 0 0 \%}$ \\
\hline
\end{tabular}

Fuente: Propia

Se evidencia que las madres que participaron en la investigación, consideraron que sus hijos se encuentran en buen estado de salud. Lo anterior es contradictorio con los indicadores de estamentos del estado que manifiestan estado de desnutrición en esta población.

Tabla 5: Estado de salud de sus hijos

\begin{tabular}{lcc}
\hline \multicolumn{1}{c}{ Opciones } & $\mathbf{N}^{\circ}$ & $\mathbf{\%}$ \\
\hline Buena & 10 & $91 \%$ \\
Mala & 1 & $9 \%$ \\
Total & $\mathbf{1 1}$ & $\mathbf{1 0 0 \%}$ \\
\hline
\end{tabular}

Fuente: Propia

De manera general se puede decir que el cuidado para las madres Wayuu se encuentra relacionado con la alimentación básica, y el cuidado de algunos aspectos de salud, pero existe desconocimiento de parte de ellas acerca de la higiene o la alimentación (Tabla 5).

Con respecto a las relaciones afectivas, entre las madres y sus hijos, se identificó lo siguiente:

Con respecto a las relaciones afectivas, entre las madres y sus hijos, se identificó lo siguiente: Las madres participantes, respondieron que algunas veces preguntan a sus hijos como se sienten cuando lo observan decaído o con el estado de ánimo bajo. Es propia de esta cultura la poca comunicación entre padres e hijos, ya que se considera que se les prepara cada día sobre la resolución de problemas en la práctica (Tabla 6). 
Tabla 6: Pregunta a su hijo(a) cómo se siente cuando lo observa decaído

\begin{tabular}{lcc}
\hline \multicolumn{1}{c}{ Opciones } & $\mathbf{N}^{\circ}$ & $\mathbf{\%}$ \\
\hline Nunca & 1 & $9 \%$ \\
Algunas veces & 7 & $64 \%$ \\
Siempre & 3 & $27 \%$ \\
Total & $\mathbf{1 1}$ & $\mathbf{1 0 0 \%}$ \\
\hline
\end{tabular}

Fuente: Propia

Las madres participantes en la investigación respondieron que juegan algunas veces con sus hijos (Tabla 7). Se considera que el joven debe ser preparado para aspectos básicos de supervivencia. El tiempo que comparten de juego es en torno a las actividades diarias de la etnia, con algunos juegos propios de la cultura.

Tabla 7: Juega con su hijo.

\begin{tabular}{lcc}
\hline \multicolumn{1}{c}{ Opciones } & $\mathbf{N}^{\circ}$ & $\mathbf{\%}$ \\
\hline Nunca & 0 & $0 \%$ \\
Algunas veces & 8 & $73 \%$ \\
Siempre & 3 & $27 \%$ \\
Total & $\mathbf{1 1}$ & $\mathbf{1 0 0} \%$ \\
\hline
\end{tabular}

\section{Fuente: Propia}

Con respecto a los estilos de crianza, se evidencian los siguientes resultados:

De manera general se observa que las relaciones afectivas están presentes en la relación madre e hijo, pero se manifiestan de manera diferente a la cultura de la población colombiana en general. Las madres son menos expresivas y están atentas a la preparación de sus hijos para enfrentar problemas cotidianos.

Con respecto a los estilos de crianza, se evidencian los siguientes resultados:

Las decisiones de castigo en la familia generalmente son tomadas por la madre (Tabla 8), lo cual corrobora que existe en la cultura Wayuu el predominio de la autoridad materna en la corrección de los hijos. 
Tabla 8: Quien castiga al hijo cuando se comporta mal

\begin{tabular}{lcc}
\hline \multicolumn{1}{c}{ Opciones } & $\mathbf{N}^{\circ}$ & $\%$ \\
\hline Madre & 7 & $64 \%$ \\
Padre & 4 & $36 \%$ \\
Total & $\mathbf{1 1}$ & $\mathbf{1 0 0 \%}$ \\
\hline
\end{tabular}

Fuente: Propia

Se observa que la mayoría de las madres de este estudio, consideraron que se encuentran educando a sus hijos siguiendo siempre la tradición de costumbres de su etnia (Tabla 9), lo que evidencia la existencia de un arraigo de la tradición ancestral en la crianza. Se puede decir que en la muestra del presente estudio existe predominio de la autoridad materna conservando los patrones de crianza que recibieron de sus propias madres.

Tabla 9: Educación de los hijos de la manera tradicional aprendida

\begin{tabular}{lcc}
\hline \multicolumn{1}{c}{ Opciones } & $\mathbf{N}^{\circ}$ & \% \\
\hline No lo hace & 1 & $9 \%$ \\
Algunas veces lo hace & 4 & $36 \%$ \\
Siempre lo hace & 6 & $54 \%$ \\
Total & $\mathbf{1 1}$ & $\mathbf{1 0 0 \%}$ \\
\hline
\end{tabular}

Fuente: Propia

Con relación a la percepción de la crianza y el trabajo, se obtuvieron los siguientes resultados:

Se evidencia que la mayoría de las madres participantes en la investigación, según la Tabla 10, no se encuentran de acuerdo en que sus hijos la acompañen a trabajar en el basurero, pero, como se señaló anteriormente, existe la necesidad de mejorar los ingresos familiares.

Tabla 10: Está de acuerdo con que sus hijos le acompañen en el trabajo de reciclaje

\begin{tabular}{lcc}
\hline \multicolumn{1}{c}{ Opciones } & $\mathbf{N}^{\circ}$ & $\%$ \\
\hline $\mathrm{Si}$ & 2 & $18 \%$ \\
$\mathrm{No}$ & 9 & $82 \%$ \\
Total & $\mathbf{1 1}$ & $\mathbf{1 0 0 \%}$ \\
\hline
\end{tabular}

Fuente: Propia 
Se observa que para 8 de las 11 madres participantes del estudio, solo algunas veces reciben colaboración voluntaria por parte de los hijos en el trabajo y 3 madres nunca la reciben de manera voluntaria por parte de sus hijos (Tabla 11). Estos resultados evidencian que los hijos colaboran de cierto modo de manera impuesta de parte de su madre, y las respuestas de las madres que indican que no estan de acuerdo pueden deberse a la deseabilidad social que muestran fnrete a los investigadores para no ser juzgadas

Tabla 11: Colaboración de manera voluntaria de parte de los hijos en el trabajo

\begin{tabular}{lcc}
\hline \multicolumn{1}{c}{ Opciones } & $\mathbf{N}^{\circ}$ & $\mathbf{\%}$ \\
\hline Nunca & 3 & $27 \%$ \\
Algunas veces & 8 & $73 \%$ \\
Siempre & 0 & $0 \%$ \\
Total & $\mathbf{1 1}$ & $\mathbf{1 0 0 \%}$ \\
\hline
\end{tabular}

Fuente: Propia

\section{Discusión}

A partir de los resultados manifiestos en la recolección de la información a través de los instrumentos aplicados a las 11 madres de menores trabajadores de la etnia Wayuu que habitan en los alrededores del basurero de la ciudad de Riohacha-La Guajira (Colombia) se evidenció un arraigo con el modelo de crianza heredado de los padres de la cultura ancestral en la etnia Wayuü, de la cual provienen. Existe predominio del mandato de la madre, donde prima la subsistencia económica que involucra a los menores en el trabajo. El entorno familiar de los menores recicladores del basurero de Riohacha se rige por principios rectores como el cultural que exige a los menores de estas familias a adoptar el rol social de aportadores, contrario a los principios rectores de las familias occidentales (arijunas); por lo que existen diferencias entre ambas culturas en cuanto a sus construcciones sociales y culturales (Monje, 2015).

Las prácticas de crianza en el menor trabajador reciclador del basurero de Riohacha, presentan tendencia a un estilo de crianza permisivo e indiferente, en donde la preocupación de la madre y de la familia es el cubrimiento de las necesidades básicas, y no de las necesidades afectivas que estos menores puedan o deban tener. En este sentido las madres participantes en el estudio no están asumiendo a plenitud la posición de proveedores y la función de educadores, a la que se refieren Rodríguez, Santos, Talani y Tovar (2014) y Pérez-Caramés (2010). En las madres de la etnia Wayuu, participantes del 
estudio, existen relaciones de afecto con sus hijos, pero como característica general se prefiere satisfacer necesidades básicas, manifestándose en la enseñanza y preparación de los hijos para la vida (Marín, 2014), al enfrentar las situaciones cotidianas de supervivencia y aprendizaje de su cultura.

La madre representa la figura de autoridad, encargándose de la toma las decisiones de castigo y de todo lo que implique asumir la crianza; muy pocas madres perciben a su esposo como un compañero que le acompañe en la educación de sus hijos. No se desconoce la autoridad masculina, sino que el esposo delega la crianza a la madre, lo cual es propio en la cultura Wayuu que concede gran importancia a la línea matriarcal (Marín, 2014). No obstante, en las madres participantes, quienes asumen la crianza de los hijos, poco se observa la figura materna que debe sostener y regular emocionalmente en la crianza, debido a que poco hablan y juegan con sus hijos, ni tampoco buscan otras opciones para el sostenimiento del hogar que no involucre el trabajo de sus hijos, a pesar de que manifiestan no estar de acuerdo con el trabajo del menor. Las madres adoptan un modelo de estilo de crianza, que de acuerdo con lo señalado por Vergara (2002), es transitorio entre madre permisiva y madre indiferente; similar a lo encontrado por investigación realizada en ciudad de Huancayo, en donde se afirma que los estilos de crianza que adoptan los padres están directamente relacionados con los riesgos de trabajo al que exponen los niños, niñas y adolescentes recicladores (Beraún, 2013).

Las madres aceptan que su hijo las acompañe, siguiendo la tradición de trabajo que tienen estas familias dedicadas al reciclaje de la basura. No existe en ellas la percepción de que sus hijos puedan contraer algún tipo de enfermedad, o puedan dedicarse a otra actividad diferente a la de acompañar a sus madres al basurero. Estos aspectos resaltan la preponderancia de la relación diádica madre-hijo propio de familias y sociedades con tendencia matriarcal como en el caso de familias campesinas, estudiadas por Luna (2002), y de las familias Wayuu (Marín, 2014). El rol que desempeña el hijo o hija Wayuu es importante para la dinámica de la familia, similar a lo encontrado en investigación realizada en la ciudad de Barranquilla por la Universidad del Norte (Amaris, Polo y Alvarez, 2008). Para la OIT (2010, p.28), "el factor determinante de la participación de miembros de los pueblos indígenas en formas de explotación económica que promueven el trabajo infantil, ha sido la perdida de sus territorios y la desvertebración de sus sociedades y culturas, por el impacto del conflicto interno y la pobreza"; esta sería otra mirada a la luz de factores relacionados con la supervivencia en la población indígena que le lleva a involucrar a todos los miembros de su familia para sobrellevar la falta de recursos económicos. 
Siendo parte de las tradiciones Wayuü que los menores de la zona trabajen en el basurero, de lo que infortunadamente, existen posibilidades de consecuencias o secuelas psicológicas, que incidirían en un desarrollo integral normal, debido a que se trunca su educación y recreación y podría ocasionar deserción escolar, por asumir desde temprana edad el papel de un adulto (Acevedo, Quejada y Yanes, 2011; Gil Batista, 2006; Cuervo, 2009; Otálvaro, 2011; Pérez-Caramés, 2010).

En el Distrito Especial Turístico y Cultural de Riohacha, este fenómeno del menor trabajador, no solo se presenta en las familias de la etnia wayuu que habitan los alrededores del basurero, también se evidencia en muchos sectores de esta ciudad, como el mercado nuevo, mercado viejo, en calles y avenidas, como ayudantes de vendedores de gasolina venezolana (pimpineros), vendedores de tinto, lavadores de autos. No solamente se evidencia en menores wayuu, también es practicado por menores de la hermana República Bolivariana de Venezuela, cuyas familias se han asilado en Colombia, ante la grave situación que atraviesa ese País. Lo cual sugiere desarrollar acciones apoyadas por el Gobierno Nacional, porque se trata de un problema que golpea duramente a la niñez sin importar nacionalidad.

\section{Conclusiones y recomendaciones}

En el análisis al entorno familiar del menor trabajador de Riohacha, desde el punto de vista sociodemográfico se encuentra que las madres en promedio oscilan entre las etapas de adultez joven y media; con familias moderadamente numerosas; la mayoría de los hijos están estudiando, los que no lo hacen es porque todavía no están en edad escolar o ya son hijos mayores. El contexto donde habitan se caracteriza por la no presencia total de grupos armados, pero si una gran ausencia del Estado y de una gran proporción de familias desplazadas y falta de oportunidades.

Tienen como principal fuente de ingresos el trabajo informal e independiente como: recicladores, vendedores de artesanías wayuü y lavadores de carro; las madres en su gran mayoría requieren la compañía de sus hijos, especialmente los menores, para realizar sus labores de trabajo, debido a que los ingresos totales incluyendo a los de los padres e hijos mayores son a veces insuficientes y no alcanzan para cubrir todas las necesidades.

Aunque se tiene acceso a servicios de salud porque todos están cobijados por el SISBEM, estos ingresos no alcanzan para cubrir completamente las necesidades de educación, vivienda aunque sea propia, y menos la de alimentación. El nivel educativo de los padres es muy bajo, escasamente la mayoría 
ha alcanzado el nivel de primaria. El tipo de familia predominante es mononuclear.

El entorno de estas familias desde el punto de vista sociodemográfico es de pobreza, presentando muchas falencias y necesidades que se convierten en un aliciente para que la mayoría permita que muchos de sus miembros menores de edad se vean obligados a trabajar.

En la identificación de las prácticas de crianza del menor trabajador de Riohacha con relación al cuidado, las relaciones afectivas, los estilos de crianza y la percepción en la crianza, se encontró lo siguiente.

- En la dimensión relaciones afectivas con los padres, existe la presencia de elementos básicos en la comunicación, lo que se ratifica con el hecho de que gran parte de las madres pregunta a sus hijos como se siente, a la mayoría les resulta fácil entender lo que sus hijos quieren o necesitan.

- También se evidencia que las madres juegan poco con sus hijos, pero la mayoría conocen la preferencia de sus hijos en cuanto juegos, lo cual es positivo. El apego se da en menor proporción, esto era de esperarse pues por las actividades laborales que limita el tiempo de atención, así como el bajo nivel educativo de las madres limita el acompañamiento en actividades escolares o académicas.

- En la dimensión estilo en la crianza de los hijos, aunque el padre representa la figura de autoridad, son las madres las que más castigan a los hijos en la mayoría de los casos. En cuanto a permisividad/ indiferencia, términos generales se encontró cierta permisividad y poca indiferencia a las actividades o acciones que realizan los hijos menores. Se puede entender en el modelo de crianza autoritario que tiene la mayoría de las madres y lo relacionado con el modelo de crianza heredado de los padres, en el arraigo cultural ancestral en la etnia Wayuü, de la cual provienen.

- En lo que respecta a la indagación acerca del concepto de trabajo que tienen las madres del menor trabajador de Riohacha. La mayor parte de ellas son conscientes que sus hijos están expuestos a algún tipo de riesgo en su seguridad al encontrarse trabajando. Gran parte de los hijos hace el acompañamiento con poca colaboración voluntaria y manifiestan alguna reacción al acompañamiento. La mayoría de las madres consideran que sus hijos pueden alternar las actividades laborales con las actividades recreativas y las actividades escolares. Lo que evidencia que los padres en gran medida están de acuerdo con el trabajo en los menores y no ven las consecuencias que esto puede acarrear o las secuelas psicológicas que puede dejar en sus hijos, como exposición a los factores de riesgos laborales, donde estos me- 
nores no cuentan con los elementos de protección personal para trabajar, así mismo, se incide en el normal desarrollo integral del menor como individuo que puede desarrollar todas sus potencialidades, pues se comienza a truncar su educación y su recreación, asumiendo desde temprana edad el papel de adulto.

Esto pone en evidencia la violación a lo establecido en la Constitución Colombiana, a la Ley 1098 de 2006, por la cual se expide el Código de la Infancia y la Adolescencia en Colombia y a la Convención Internacional de los Derechos del Niño, en lo que tiene que ver con los derechos de los menores y adolescentes, lo cual requiere una pronta intervención del Estado.

A partir de las conclusiones obtenidas se hacen las siguientes recomendaciones:

- Profundizar aún más en el análisis del entorno familiar del menor trabajador, en especial de las familias que derivan su sustento de trabajos informales, y la incidencia real que esto trae para el desarrollo cognitivo e integral de los menores trabajadores.

- Enfocar las teorías de entorno familiar y prácticas de crianza para experimentar y perfeccionar modelos que no sean compatibles con el trabajo infantil y así erradicar este flagelo de una sociedad indiferente. Que promueva que los menores vayan a la escuela y no deserten de ella por trabajar, que ocupen sus ratos libres en la sana diversión, el juego y la recreación que son actividades que favorecen su desarrollo integralmente.

- Promover la intervención de familias en un acompañamiento conformado por un grupo interdisciplinar, que permita, respetando los aspectos culturales de estas familias, sobre todo los de tipo étnico, ir introduciendo las mejoras en las prácticas de crianza.

- Llamar la atención y hacer que las comunidades conozcan y cumplan lo contemplado en la Constitución Política de Colombia y la Convención Internacional de los derechos del niño, que recogen los principios donde establecen las diferentes perspectivas sobre derechos de los niños y niñas, como sujetos activos de unos derechos básicos fundamentales, universales e indivisibles, los cuales, a su vez, generan deberes ineludibles para el Estado y la sociedad, quienes deben corresponder y garantizar estos derechos considerando el interés superior de niños y niñas en todas sus decisiones. 


\section{Referencias}

Acevedo, K., Quejada R., Yanez, M.(2011). Determinantes y consecuencias del trabajo infantil. Un análisis de la literatura. Revista de la Facultad de Ciencias Económicas de la Universidad Militar Nueva Granada, 19(1): 113-124. Disponible en http://www.scielo.org.co/pdf/rfce/v19n1/v19n1a07.pdf

Amaris, M., Polo, J., Alvarez, M. (2008). Rol del menor en su familia. Salud Uninorte, 24 (1): 87-97. Disponible en http://rcientificas.uninorte.edu.co/index.php/ salud/article/viewArticle/3821/5742

Beraún, H. (2013). Estilos de crianza y los riesgos del trabajo de los niños, niñas y adolecentes recicladores de basura de la ciudad de Huancayo - 2012. Convicciones, 1(1): 76-83. Disponible en http://repositorio.uncp.edu.pe/handle/UNCP/826

Congreso de Colombia, (2006). Ley 1098 de 2006, Por la cual se expide el Código de la Infancia y la Adolescencia. Bogotá D.C. Disponible en https://www.oas.org/dil/ esp/Codigo_de_la_Infancia_y_la_Adolescencia_Colombia.pdf

Congreso de Colombia, (2012). Ley 1581 de 2012, Por la cual se dictan disposiciones generales para la protección de datos personales. Bogotá D.C. Disponible en http:// www.alcaldiabogota.gov.co/sisjur/normas/Norma1.jsp? $\mathrm{i}=49981$

Coria, A., Jasso, B., Paz, E., Nájera, P. (2012). El entorno familiar y su influiencia en el plan de vida de los jovenes en una universidad privada de Monterrey, Mexico. Intersticios: revista sociológica de pensamientos críticos, 6(2): 175-190. Disponible en http://www.intersticios.es/article/view/10460

Cuervo, A. (2009). Pautas de crianza y desarrollo socioafectivo en la infancia. Revista Diversitas, Perspectivas en psicología, 6(1): 111-121. Disponible en http://www. scielo.org.co/pdf/dpp/v6n1/v6n1a09.pdf

DANE (2016). Mercado laboral trabajo infantil. Disponible en http://www.dane.gov. co/index.php/estadisticas-por-tema/mercado-laboral/trabajo-infantil

Erikson, E. (2000). El ciclo vital completado. Buenos Aires: Ediciones Paidós.

Gil Batista, A. (2006). Análisis del trabajo infantl en Colombia:Perspectiva legal y psicológica. Bogotá D.C.: ESAP - Facultad de Postgrado Especialización en derechos humanos. Disponible en http://cdim.esap.edu.co/BancoMedios/Documentos\%20 PDF/an\%C3\%A1lisis\%20del\%20trabajo\%20infantil\%20en\%20colombia\%20 perspectiva\%20legal\%20y\%20psicol\%C3\%B3gica.pdf

Gonzalez, A., Pimienta, I. (2004). Estudio socio juridico del menor trabajador en Colombia. Pontificia Universidad Javeriana. Trabajo de grado, Facultad de Ciencias 
juridicas, Universidad Pontificia Javeriana, Bogota D.C. Disponible en https:// www.javeriana.edu.co/biblos/tesis/derecho/dere6/DEFINITIVA/TESIS42.pdf

OIT (2010). Trabajo Infantil Indígena en Colombia. Una síntesis de las miradas sobre el problema desde las comunidades indígenas, los académicos y las instituciones. Disponible en Web: www.oit.org.pe/ipec

OIT (2017). Estimaciones Mundiales sobre el trabajo infantil. Resultados y tendencias 2012-2016. Disponible en https://www.ilo.org/wcmsp5/groups/public/@ed_ norm/@ipec/documents/publication/wcms_596481.pdf

Otálvaro, J. (2011). La crianza del niño trabajador: una reflexión desde la salud pública. Revista Facultad Nacional de Salud Pública, 29 (4): 495-503. Disponible en https://aprendeenlinea.udea.edu.co/revistas/index.php/fnsp/article/view/9688

Luna, M. (2002). Prácticas de crianza en Antioquia, un estudio de familias campesinas. Medellín: CINDE. Disponible en: https://es.scribd.com/document/49893670/ Practicas-de-Crianza-en-Antioquia\#

Marín, M. (2014). Cosmogonía y Rito en la Vivienda Wayuu. Universidad Nacional de Colombia Facultad de Ingeniería y Arquitectura Escuela de Arquitectura y Urbanismo Manizales, Colombia.

Monje, J. (2015). El plan de vida de los pueblos indígenas de Colombia, una construcción de etnoecodesarrollo. Revista Luna Azul, 41: 29-56, DOI: 10.17151/ luaz.2015.41.3

Pérez-Caramés, A. (2010). Configuraciones del trabajo de cuidados en el entorno famliar. Dela toma de decisiones a la gestión del cuidado. Revista Alternativas. Cuadernos de Trabajo Social, 17, 121-140. Doi 10.14198/ALTERN2010.17.7

Rodríguez, M., Santos, C., Talani, J., Tovar. (2014). Prácticas y creencias culturales acerca del cuidado de niños menores de un año en un grupo de madres de Chocontá, Colombia. Revista Colombiana de enfermería, 9 (9): 77-87. Disponible en http://m.uelbosque.edu.co/sites/default/files/publicaciones/revistas/revista colombiana_enfermeria/volumen9/010-articulo8.pdf

Schaie, K. W., Willis, S. (2016). Manual de la psicología del envejecimiento. San Diego: Academic Press.

Torres, M. (2009). Prácticas de crianza y educación inicial en niños Mayo / Yoreme. Hermosillo, Sonora. México: Centro de investigación en alimentación y desarrollo A.C. Disponible en http://www.ciad.mx/archivos/desarrollo/publicaciones/ Tesis\%20asesoradas/Tesis\%20Maestria/30.pdf 
Vergara, C. (2002). Creencias relacionadas con las prácticas de crianza de los hijos. México: Universidad de Colma. Disponible en http://digeset.ucol.mx/tesis posgrado/Pdf/Claudia\%20Berenice\%20Vergara\%20Hernandez.pdf

Viguer, P., Serra, E. (1996). Nivel socioeconomico y calidad del entorno famiiliar en la infancia. Anales de psicología, 12 (2) 197 - 205. Disponible en https://www. um.es/analesps/v12/v12_2/08-12-2.pdf 